\title{
PAX6 overexpression is associated with the poor prognosis of invasive ductal breast cancer
}

\author{
XIANGHOU XIA ${ }^{1}$, WENJUAN YIN ${ }^{2}$, XIPING ZHANG $^{1}$, XINGFEI YU $^{1}$, CHEN WANG $^{1}$, \\ SHENHUA $\mathrm{XU}^{3}$, WEILIANG FENG ${ }^{1}$ and HONGJIAN YANG ${ }^{1,4}$ \\ Departments of ${ }^{1}$ Breast Surgery and ${ }^{2}$ Pathology, Zhejiang Cancer Hospital; ${ }^{3}$ Zhejiang Cancer Research Institute, \\ Hangzhou, Zhejiang; ${ }^{4}$ Department of Surgical Oncology, School of the First Clinical Medical Sciences, \\ Wenzhou Medical University, Wenzhou, Zhejiang, P.R. China
}

Received October 14, 2014; Accepted April 28, 2015

DOI: $10.3892 / 01.2015 .3434$

\begin{abstract}
Paired box 6 (PAX6) plays a significant role in the development of human neuroectodermal epithelial tissues. Previous studies have suggested that the PAX6 promoter is hypermethylated in breast cancer and that it is involved in breast cancer cell proliferation. The present study aimed to investigate the expression of PAX6 in invasive breast cancer tissues, and to evaluate its prognostic significance. Immunohistochemistry (IHC) was used to detect PAX6 expression on a breast cancer tissue microarray containing tissues from 111 patients. Associations of PAX6 expression with staging and prognosis were analyzed. PAX6 was mainly expressed in the nucleus. The PAX6 staining intensity was not associated with age, histological grade, lymph node status, tumor size, or progesterone receptor and human epidermal growth factor receptor 2 expression (all $\mathrm{P}>0.05$ ). A high level of PAX6 staining was more frequent in estrogen receptor (ER)-negative cases compared with ER-positive cases (43.9 vs. 25.7\%; $\mathrm{P}=0.049$ ). After a median follow-up time of 110 months, the patients with low PAX6 expression exhibited an improved survival rate compared with the patients with high PAX6 expression $(\mathrm{P}<0.001)$. Cox analysis showed a worse survival rate in the patients with high PAX6 staining (hazard ratio, 3.458; 95\% confidence interval, 1.575-7.593; P=0.002). In conclusion, high tumor PAX6 staining intensity by IHC was associated with a poor prognosis in breast cancer patients.
\end{abstract}

Correspondence to: Dr Hongjian Yang, Department of Surgical Oncology, School of the First Clinical Medical Sciences, Wenzhou Medical University, 2 Fuxue Road, Wenzhou, Zhejiang 325000, P.R. China

E-mail: hongjianyangsci@126.com

Key words: breast cancer, paired box 6, prognosis, human epidermal growth factor receptor 2, progesterone receptor, estrogen receptor

\section{Introduction}

Breast cancer is a major health concern worldwide. In the United States, the breast cancer incidence is 120.7 per 100,000 women and breast cancer mortality is 24 per 100,000 women; the lifetime risk of breast cancer is $12.2 \%$ (1). The breast cancer incidence in China is 3.5 times lower than in the United States (2), but models strongly suggest that breast cancer will soon reach epidemic proportions in China (3). Invasive ductal carcinomas represent about $\sim 75 \%$ of all breast cancers (4). A number of lifestyle habits are associated with the risk of developing breast cancer (5), and recent changes in Chinese lifestyle, such as a shift toward a Western diet and increased stress, may explain a part of this increase (6). Our understanding of the pathogenesis of breast cancer has gaps $(7,8)$, and there is an urgent requirement to improve this knowledge.

The paired box (PAX) family member, PAX6, is located on chromosome 11p13 in humans, and encodes the PAX6 protein that plays an important role in the development of human neuroectodermal epithelial tissues (9). A previous study reported that PAX6 participates in the regulation of neuroectodermal cell differentiation and apoptosis (10). PAX6 has also been suggested as a tumor suppressor gene for glioblastoma and prostate cancer, and as an early differentiation marker for neuroendocrine cells (11).

PAX genes are often expressed in solid cancers and are necessary for cancer cell survival (12). In prostate cancer tissues, the expression level of PAX6 in cancer cells is significantly lower than in normal epithelial cells (11). An epigenetic study revealed that the promoter of PAX6 was hypermethylated in breast cancer (13). Our previous study revealed that PAX6 is involved in breast cancer cell proliferation and tumor progression in vitro (14), while a screening of cancer cell lines revealed that PAX6 was highly expressed in breast cancer cell lines (12).

However, to the best of our knowledge, no study has yet detected the expression of PAX6 in invasive ductal breast cancer tissues and evaluated its prognostic significance. The present study aimed to investigate PAX6 protein expression in an invasive ductal breast cancer tissue microarray (TMA) using immunohistochemistry (IHC), and to 
evaluate the association between PAX6 expression and the prognosis of invasive ductal breast cancer, in order to show the potential of PAX6 for improving the assessment of invasive ductal breast cancer prognosis and as an eventual treatment target.

\section{Subjects and methods}

Human subjects and tissue specimens. The cohort included a total of 119 patients with invasive ductal breast cancer, diagnosed and surgically treated between January 2001 and December 2003 at the Department of Breast Cancer, Zhejiang Cancer Hospital (Hangzhou, Zhejiang, China). All patients had received no radiotherapy or neoadjuvant therapy prior to surgery. Of these 119 cases, 8 patients were excluded due to incomplete medical records. The remaining 111 patients with primary breast cancer received conventional post-operative treatments, depending on the extent of the disease. Patients without axillary lymph node involvement were treated with a modified radical mastectomy and 6 cycles of cyclophosphamide $\left(100 \mathrm{mg} / \mathrm{m}^{2}\right.$, orally, days $\left.1-14\right)$, methotrexate $\left(40 \mathrm{mg} / \mathrm{m}^{2}\right.$, days 1 and 8$)$ and 5 -fluorouracil $\left(600 \mathrm{mg} / \mathrm{m}^{2}\right.$, days 1 and 8 ) chemotherapy, cycled every 28 days (if the tumor size was $\geq 1 \mathrm{~cm}$ ). Patients with axillary lymph node involvement received 5-fluorouracil $\left(500 \mathrm{mg} / \mathrm{m}^{2}\right.$, day 1$)$, epirubicin $\left(100 \mathrm{mg} / \mathrm{m}^{2}\right.$, day 1) and cyclophosphamide $\left(500 \mathrm{mg} / \mathrm{m}^{2}\right.$, day 1) chemotherapy, cycled every 21 days for 3 cycles, followed by docetaxel chemotherapy $\left(100 \mathrm{mg} / \mathrm{m}^{2}\right.$, day 1$)$, cycled every 21 days for 3 cycles. Alternatively, certain patients received epirubicin $\left(100 \mathrm{mg} / \mathrm{m}^{2}\right.$, day 1$)$ and cyclophosphamide $\left(500 \mathrm{mg} / \mathrm{m}^{2}\right.$, day 1) chemotherapy, cycled every 21 days for 4 cycles, followed by docetaxel chemotherapy $\left(100 \mathrm{mg} / \mathrm{m}^{2}\right.$, day 1$)$, cycled every 21 days for 4 cycles. Patients with positive nodes or a tumor size of $\geq 5 \mathrm{~cm}$ received postoperative radiotherapy to the breast (50 Gy in 25 fractions). A boost to the tumor bed was applied in patients at higher risk (those aged $<50$ years and those with high-grade disease). Patients with estrogen receptor $(E R)^{+} /$progesterone receptor $(\mathrm{PR})^{+}$tumors were treated for 5 years with tamoxifen or aromatase inhibitors. Patient characteristics, including age, menopausal status and clinical stage (tumor-node-metastasis classification defined by the International Union against Cancer, 2003) (15), were assessed.

All patients were followed up for at least 4 months, and up to 131 months or until mortality. Patients who were lost to follow-up due to mortality or any other reasons were censored at last contact. Overall survival (OS) time was calculated as the time between surgery and mortality from any cause. This study was approved by the Clinical Research Ethics Board of the Zhejiang Cancer Hospital. Written informed consent was obtained from each patient.

TMAs and IHC. TMAs were constructed using a Beecher Instruments Tissue Array (Beecher Instruments, Silver Spring, MD, USA) (16). Briefly, archival paraffin blocks containing invasive ductal carcinoma tissues were selected. Two cores of $1.5 \mathrm{~mm}$ in diameter were sampled from the tumor area of each specimen (donor block), and transferred into the TMA block (recipient block). Consecutive 4- $\mu \mathrm{m}$ thick sections were cut from the TMA blocks and placed on a poly-L-lysine-coated slide for IHC analysis. Antigens were retrieved for $5 \mathrm{~min}$ in a microwave at high power followed by $10 \mathrm{~min}$ at low power in citrate-buffered saline (pH 6.0). ER, PR, human epidermal growth factor receptor 2 (HER2) and PAX6 were detected by IHC. The following antibodies were used: mouse monoclonal anti-PAX6 (clone D2.38; cat. no. ab78545; 1:150 dilution; Abcam, Cambridge, MA, USA), rabbit monoclonal anti-ER (clone SP1; cat. no. ab16660; 1:100 dilution; Abcam), mouse monoclonal anti-PR (clone IA6; cat. no. M3569; 1:200 dilution; Dako, Carpinteria, CA, USA), and rabbit polyclonal anti-HER2/neu (clone SP3; cat. no. ab2428; 1:100 dilution; Abcam). Developing human brain tissues (paraffin-embedded frontal cortex tissues from children with glioblastoma who had undergone biopsy at the hospital) known to express high levels of PAX6 were used as the positive control. Samples from patients with cancers known to be HER2-amplified or with ER/PR overexpression were used as positive controls for HER2, ER and PR. Negative controls were performed by omission of the primary antibody.

$E R, P R$ and HER2 scoring. TMA was scored by two pathologists for the percentage of tumor cell nuclear positivity. ER and PR were scored as follows:,$-<1 \% ; 1+, 1-25 \% ; 2+, 25-75 \%$; or $3+$, $>75 \%$. IHC ER and PR scores were dichotomized as follows: 0 , negative; and $\geq 1+$, positive. HER 2 was scored as: 0 , no staining or faint membrane staining; $1+$, faint membrane staining in $<10 \%$ of tumor cells, incomplete membrane staining; $2+$, weak to moderate membrane staining in $>10 \%$ of tumor cells; and $3+$, strong complete membrane staining in $>10 \%$ of tumor cells. IHC HER2 scores were considered negative at $0 / 1+$. All samples with a HER2 score of 2+ were tested for gene amplification by fluorescence in situ hybridization (FISH; Vysis PathVysion; Abbott Laboratories, Chicago, IL, USA). Slides were hybridized with probes to HER-2/neu and CEP17 using the PathVysion HER-2 DNA Probe Kit, according to the manufacturer's instructions. Sections were counterstained with DAPI and visualized on a fluorescent microscope (DM4000B, Leica Biosystems Nussloch $\mathrm{GmbH}$, Nussloch, Germany). Scoring was performed by one pathologist according to the manufacturer's guidelines, yielding a HER-2/CEP17 ratio. A HER-2/CEP17 ratio of $\geq 2$ was considered amplified. IHC HER2 scores of 3+ and FISH-amplified patients were considered positive. Pathologists were blinded to the clinical outcomes.

PAX6 scoring. The IHC expression of PAX6 was scored independently using a semi-quantitative scoring system by two pathologists who were blinded to the characteristics and outcomes of the patients. Discordant scores were re-evaluated by the investigators and consensus scores were used for further analyses. The intensity and the extent of IHC staining were assessed. Staining intensity in the nucleus was defined as follows: No staining, 0 points; light brown particles, 1 point; moderate brown particles, 2 points; and dark brown particles, 3 points. The percentage of positive cells was scored as: $1,<25 \%$ positive cells; $2,25-50 \%$ positive cells; $3,51-75 \%$ positive cells; and $4,>75 \%$ positive cells. The staining index (SI) was calculated as the product of the intensity and the percentage of positive staining, and PAX6 expression was defined as high $(\mathrm{SI} \geq 6)$ or low $(\mathrm{SI}<6)$.

Statistical analysis. Statistical analyses were performed using SPSS 19.0 (IBM, Armonk, NY, USA). Correlations between PAX6 expression and the clinicopathological variables were 
Table I. Association between PAX6 expression and the clinicopathological features of invasive ductal breast cancer in 111 patients.

\begin{tabular}{|c|c|c|c|c|}
\hline \multirow[b]{2}{*}{ Variables } & \multirow[b]{2}{*}{ Total, n (\%) } & \multicolumn{2}{|c|}{ PAX6 expression, n (\%) } & \multirow[b]{2}{*}{ P-values } \\
\hline & & Low $(n=75)$ & $\operatorname{High}(\mathrm{n}=36)$ & \\
\hline \multicolumn{5}{|l|}{ Age, years } \\
\hline$<50$ & $58(52.3)$ & $40(69.0)$ & $18(31.0)$ & \multirow[t]{3}{*}{0.938} \\
\hline $50-69$ & 39 (35.1) & $25(64.1)$ & 14 (35.9) & \\
\hline$>70$ & $14(12.6)$ & $10(71.4)$ & $4(28.6)$ & \\
\hline \multicolumn{5}{|l|}{ Histological grade } \\
\hline G1 & $37(33.3)$ & $28(75.7)$ & $9(24.3)$ & \multirow[t]{3}{*}{0.431} \\
\hline $\mathrm{G} 2$ & $71(34.0)$ & $44(62.0)$ & $27(38.0)$ & \\
\hline G3 & $3(2.7)$ & $3(100.0)$ & $0(0.0)$ & \\
\hline \multicolumn{5}{|l|}{ Lymph node status } \\
\hline No & $42(37.9)$ & $25(59.5)$ & $17(40.5)$ & \multirow[t]{4}{*}{0.063} \\
\hline N1 & $36(32.4)$ & $24(66.7)$ & $12(33.3)$ & \\
\hline $\mathrm{N} 2$ & $25(22.5)$ & $19(76.0)$ & $6(24.0)$ & \\
\hline N3 & $8(7.2)$ & $7(87.5)$ & $1(12.5)$ & \\
\hline \multicolumn{5}{|l|}{ Tumor size, $\mathrm{cm}$} \\
\hline 2 & $21(18.9)$ & $15(71.4)$ & $6(28.6)$ & \multirow[t]{3}{*}{0.976} \\
\hline $2-5$ & $78(70.3)$ & $51(65.4)$ & $27(34.6)$ & \\
\hline$>5$ & $12(10.8)$ & $9(75.0)$ & $3(25.0)$ & \\
\hline \multicolumn{5}{|l|}{ ER } \\
\hline Negative & $41(36.9)$ & $23(56.1)$ & $18(43.9)$ & \multirow[t]{2}{*}{0.049} \\
\hline Positive & $70(63.1)$ & $52(74.3)$ & $18(25.7)$ & \\
\hline \multicolumn{5}{|l|}{ PR } \\
\hline Negative & $49(44.1)$ & $32(65.3)$ & $17(34.7)$ & \multirow[t]{2}{*}{0.654} \\
\hline Positive & $62(55.9)$ & $43(69.4)$ & $19(30.6)$ & \\
\hline \multicolumn{5}{|l|}{ HER2 } \\
\hline Negative & $99(89.2)$ & $68(68.7)$ & $31(31.3)$ & \multirow[t]{2}{*}{0.930} \\
\hline Positive & $12(10.8)$ & $7(58.3)$ & $5(41.7)$ & \\
\hline \multicolumn{5}{|l|}{ TNBC } \\
\hline No & $83(74.8)$ & $60(72.3)$ & $23(27.7)$ & \multirow[t]{2}{*}{0.101} \\
\hline Yes & $28(25.2)$ & $15(53.6)$ & $13(46.4)$ & \\
\hline \multicolumn{5}{|l|}{ Menopausal status } \\
\hline Premenopausal & $53(47.7)$ & $38(71.7)$ & $15(28.3)$ & \multirow[t]{2}{*}{0.084} \\
\hline Postmenopausal & $58(52.3)$ & $37(67.6)$ & $21(32.4)$ & \\
\hline
\end{tabular}

Staining index (SI) was calculated as the product of the intensity and the percentage of positive staining. PAX6 expression was defined as high $(\mathrm{SI}>6)$ or low $(\mathrm{SI}<6)$. PAX6, paired box 6; ER, estrogen receptor; PR, progesterone receptor; HER2, human epidermal growth factor 2; TNBC, triple-negative breast cancer.

analyzed using the Pearson $\chi^{2}$ analysis. Survival was analyzed using the Kaplan-Meier method, and differences were evaluated using the log-rank test. The Cox proportional hazards model was used for univariate and multivariate analyses to examine the potential prognostic value of different variables on OS. All tests were two-sided. $\mathrm{P}<0.05$ was considered to indicate a statistically significant difference.

\section{Results}

Patient characteristics. Table I presents the clinical characteristics of the patients with invasive ductal carcinoma of the breast. At the time of surgery, the median age was 50 years (range, 29-82 years). The histological grade of the tumor was grade I in $37(33.3 \%)$ patients, grade II in 71 (64.0) patients and grade III in $3(2.7 \%)$ patients. Lymph node status was N0 in $42(37.8 \%)$ patients, N1 in $36(32.5 \%), \mathrm{N} 2$ in $25(22.5 \%)$ and $\mathrm{N} 3$ in $8(7.2 \%)$. Tumor size was $<2 \mathrm{~cm}$ in $21(18.9 \%)$ patients, $2-5 \mathrm{~cm}$ in $78(70.3 \%)$ and $>5 \mathrm{~cm}$ in $12(10.8 \%)$. ER was positive in $63.1 \%$ of tumors, PR was positive in $55.9 \%$, HER 2 was positive in $10.8 \%$, and ER, PR and HER2 were all negative in $25.2 \%$.

PAX6 staining. PAX6 was mainly expressed in the nucleus. PAX6 was expressed at a low $(\mathrm{SI}<6$; Fig. 1A) or high $(\mathrm{SI}>6$, 
Table II. Cox proportional hazards model was used for univariate and multivariate analysis to examine the potential prognostic value of different variables on cumulative survival in the invasive ductal breast cancer patients.

\begin{tabular}{|c|c|c|c|c|}
\hline \multirow[b]{2}{*}{ Variable } & \multicolumn{2}{|c|}{ Univariable analysis } & \multicolumn{2}{|c|}{ Multivariable analysis } \\
\hline & HR $(95 \%$ CI $)$ & P-value & HR $(95 \% \mathrm{CI})$ & P-value \\
\hline PAX6 (high vs. low) & $3.246(1.610-6.539)$ & 0.001 & $3.458(1.575-7.593)$ & 0.002 \\
\hline ER (positive vs. negative) & $0.434(0.219-0.869)$ & 0.018 & $5.140(0.343-7.014)$ & 0.236 \\
\hline PR (positive vs. negative) & $0.355(0.171-0.737)$ & 0.005 & $0.298(0.089-1.004)$ & 0.051 \\
\hline TNBC (yes vs. no) & $2.773(1.383-5.583)$ & 0.004 & $1.665(0.637-4.301)$ & 0.301 \\
\hline HER2 (positive vs. negative) & $1.257(0.484-3.265)$ & 0.638 & & \\
\hline Histological grade (G2+G3 vs. G1) & $1.096(0.579-2.315)$ & 0.810 & & \\
\hline Tumor size ( $>5$ vs. $\leq 5 \mathrm{~cm})$ & $1.841(0.654-5.252)$ & 0.254 & & \\
\hline Lymph node status $(\mathrm{N} 1+\mathrm{N} 2+\mathrm{N} 3$ vs. N0) & $1.204(0.838-1.729)$ & 0.316 & & \\
\hline Menopausal status (pre- vs. post-) & $1.310(0.646-2.654)$ & 0.454 & & \\
\hline
\end{tabular}

PAX6, paired box 6; ER, estrogen receptors; PR, progesterone receptors; HER2, human epidermal growth factor 2; TNBC, triple negative breast cancer; HR, hazard ratio; CI, confidence interval.

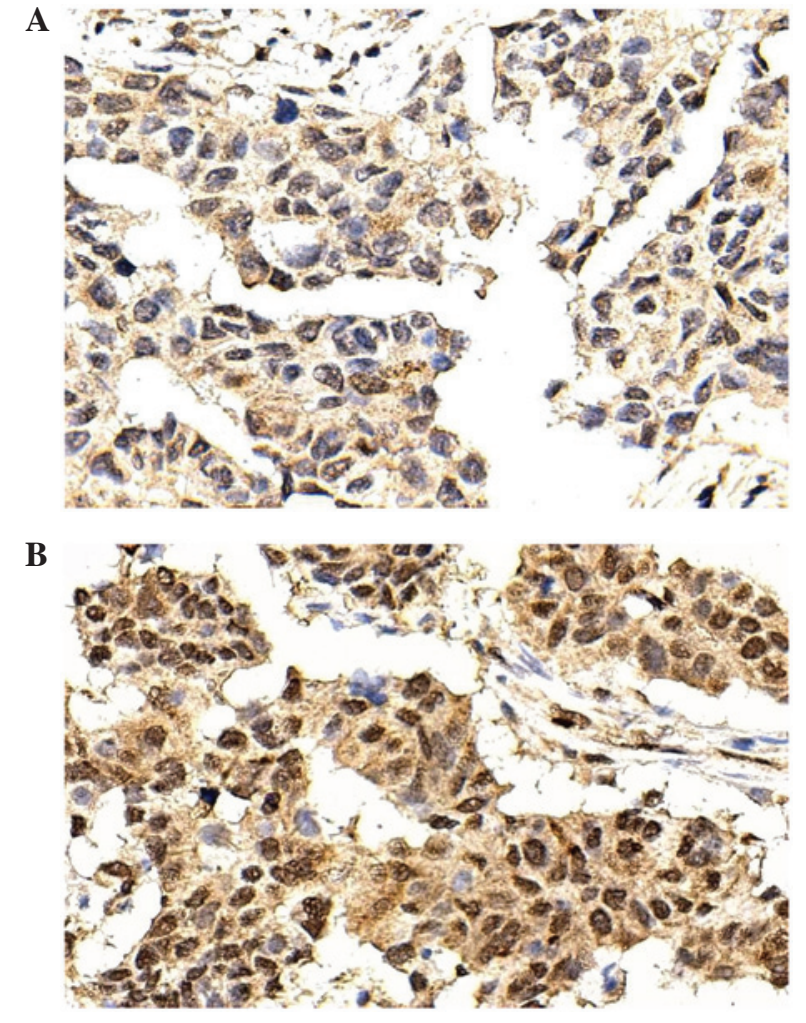

Figure 1. Expression of PAX6 in the invasive ductal breast cancer tissues, as detected by immunohistochemistry. PAX6 was mainly expressed in the nucleus. (A) Low expression ( $\mathrm{SI}<6$ ) of PAX6 in invasive ductal breast cancer tissues. (B) High expression ( $\mathrm{SI}>6$ ) of PAX6 in invasive ductal breast cancer tissues. Magnification, x400. PAX6, paired box 6; SI, staining index.

Fig. 1B) level. According to the SI, $75(67.6 \%)$ patients exhibited low PAX6 expression, and 36 (32.4\%) exhibited high PAX6 expression.

Association between PAX6 and clinicopathological characteristics. High PAX6 expression was associated with a lower proportion of ER positivity (25.7 vs. $74.3 \%$; $\mathrm{P}=0.049)$

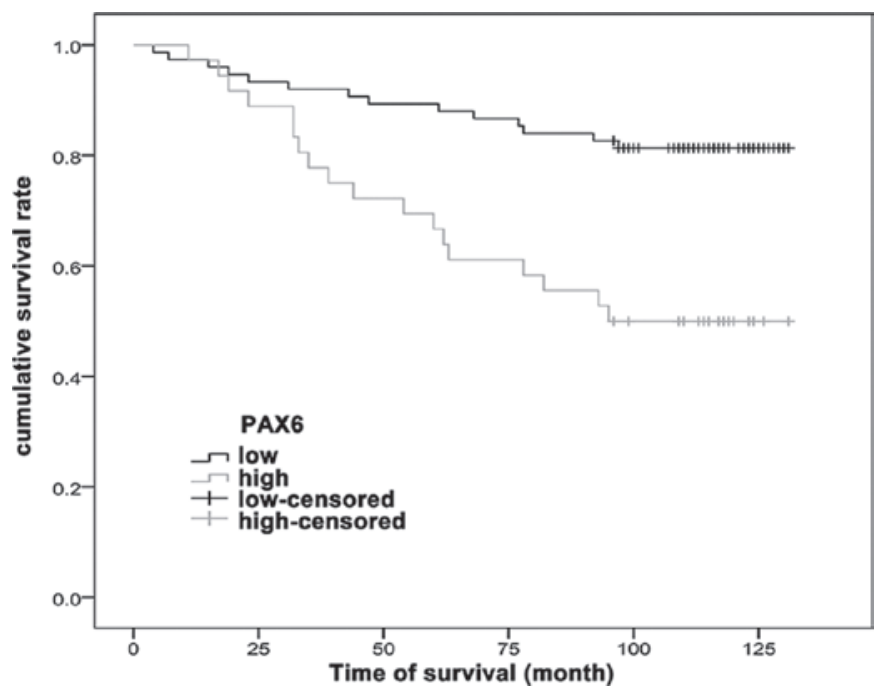

Figure 2. Kaplan-Meier curves of cumulative survival rate of invasive ductal breast cancer patients based on the PAX6 staining. Association between PAX6 staining intensity [low expression $(\mathrm{SI}<6)$ and high expression $(\mathrm{SI}>6)$ ] and cumulative survival (log-rank test; $\mathrm{P}<0.001$ ). PAX6, paired box 6; SI, staining index.

(Table I). PAX6 expression was not associated with age, histological grade, lymph node status, tumor size, PR, HER2 and triple-negative breast cancer (TNBC) (all $\mathrm{P}>0.05$ ).

Survival. After a median follow-up time of 110 months, the patients with low PAX6 expression exhibited an improved survival rate compared with the patients with high PAX6 expression $(\mathrm{P}<0.001)$ (Fig. 2).

Cox regression analysis between clinicopathological factors and survival. Univariate Cox regression analysis revealed that high PAX6 expression [hazard ratio (HR), 3.246; 95\% confidence interval (CI), 1.610-6.539; $\mathrm{P}=0.001]$, ER positivity (HR, 0.434; 95\% CI, 0.219-0.869; $\mathrm{P}=0.018$ ), PR positivity (HR, 0.355; 95\% CI, 0.171-0.737; $\mathrm{P}=0.005)$ and TNBC (HR, 2.773; 95\% CI, 1.383-5.583; $\mathrm{P}=0.004)$ were associated 
with survival. HER2, histological grade, tumor size, lymph node status and menopausal status were not associated with survival (all $\mathrm{P}>0.05$ ) (Table II).

Multivariate Cox regression analysis showed that high PAX6 expression (HR, 3.458; 95\% CI, 1.575-7.593; $\mathrm{P}=0.002)$ was independently associated with survival. Additionally, there was a tendency toward an association between PR and survival, but this was not statistically significant $(\mathrm{P}=0.051)$ (Table II).

\section{Discussion}

Previous studies have suggested a possible association between PAX6 and breast cancer. However, no previous study has investigated PAX6 expression in breast cancer according to IHC and its association with prognosis. Therefore, the present study aimed to investigate PAX6 protein expression in a breast cancer TMA using IHC, and to evaluate the association between PAX6 expression and the breast cancer prognosis.

The results showed that PAX6 staining intensity was not associated with histological grade, lymph node status, tumor size, PR or HER2. High PAX6 staining was more frequent in ER-negative cases compared with ER-positive cases. After a median follow-up time of 110 months, patients with low PAX6 expression exhibited an improved survival rate compared with patients with high PAX6 expression. Cox analysis showed a worst survival rate in patients with high PAX6 staining (HR, 3.458; 95\% CI, 1.575-7.593). Results suggest that high PAX6 expression in breast cancer is associated with a worse prognosis. PAX6 may be used to improve the assessment of breast cancer prognosis, and may eventually be a treatment target.

Shyr et al (11) used IHC to show that the PAX6 expression level in normal prostate epithelial cells was higher than that in prostate cancer cells. The results of the present study showed that PAX6 was expressed in the normal tissues bordering the tumor and in the breast cancer cells, and that the staining intensity varied between patients within the range from $2+$ to $3+$. A previous study (9) suggested that PAX6 was mainly expressed in stem cells and progenitor cells, and that PAX6 activation could lead to mitotic arrest, premature neurogenesis and apoptosis. This may explain the finding of the present study that patients with high PAX6 expression only had a survival rate of 50\%, suggesting that high PAX6 expression may be an indicator of the poor prognosis of breast cancer. Lang et al (17) suggested that PAX6 could be used as a molecular target in cancer therapies.

The present results suggested that PAX6 was an independent prognostic factor. To the best of our knowledge, this is the first study suggesting this association in breast cancer, and no other study is available for direct comparison. A previous study in gastric cancer showed that methylation of the PAX6 promoter was associated with reduced survival (18). Another study in gastric cancer showed that PAX6 was associated with markers of a poor prognosis, such as lymph node metastasis (19). In addition, PAX6 was upregulated in alveolar soft part sarcoma, a type of cancer with an extremely poor prognosis (20). In pancreatic cancer, PAX6 actively participates in tumor growth via the MET tyrosine kinase (21). However, certain studies have suggested that PAX6 may have a tumor suppressor effect, such as in glioblastoma $(22,23)$. Therefore, further studies are necessary to assess the role of PAX6 in tumorigenesis. Nevertheless, our previous in vitro study showed that PAX suppression significantly decreased cell viability, DNA synthesis and the colony formation of MCF-7 and MDA-MB-231 cells, and inhibited tumorigenesis in xenograft nude mice (14). However, another study showed that the promoter of PAX6 was frequently hypermethylated in breast cancer (13), adding to the controversy.

The present results showed that PR-negative staining may be a factor for a poor prognosis in breast cancer. There are few studies reporting PR-negative staining as an independent factor for breast cancer poor prognosis. Only Chen et al (24) suggested that no PR expression was an independent factor for the early recurrence of breast cancer, which is consistent with the present results. However, PR-negative patients include patients with certain triple-negative cancers, which may cause this difference. Further studies are required to assess this point.

The present study is not without limitation. First, the sample size was small. Second, even though the follow-up was long (median, 110 months), the survival time of breast cancer patients is generally good and a longer period may be necessary to more precisely observe the effect of PAX6 expression on prognosis.

In conclusion, a high tumor PAX6 staining intensity, as observed by IHC, was independently associated with a poor prognosis in the breast cancer patients of the present study. PAX6 may be a novel prognosis marker, and may eventually be tested as a target for therapy.

\section{References}

1. Kohler BA, Ward E, McCarthy BJ, et al: Annual report to the nation on the status of cancer, 1975-2007, featuring tumors of the brain and other nervous system. J Natl Cancer Inst 103: 714-736, 2011.

2. Wang YC, Wei LJ, Liu JT, Li SX and Wang QS: Comparison of cancer incidence between China and the USA. Cancer Biol Med 9: 128-132, 2012.

3. Linos E, Spanos D, Rosner BA, Linos K, Hesketh T, Qu JD, Gao YT, Zheng W and Colditz GA: Effects of reproductive and demographic changes on breast cancer incidence in China: A modeling analysis. J Natl Cancer Inst 100: 1352-1360, 2008.

4. Li CI, Anderson BO, Daling JR and Moe RE: Trends in incidence rates of invasive lobular and ductal breast carcinoma. JAMA 289: $1421-1424,2003$.

5. McPherson K, Steel CM and Dixon JM: ABC of breast diseases. Breast cancer-epidemiology, risk factors, and genetics. BMJ 321: 624-628, 2000.

6. Chia KS, Reilly M, Tan CS, Lee J, Pawitan Y, Adami HO, Hall P and Mow B: Profound changes in breast cancer incidence may reflect changes into a Westernized lifestyle: A comparative population-based study in Singapore and Sweden. Int J Cancer 113: 302-306, 2005.

7. Yager JD and Davidson NE: Estrogen carcinogenesis in breast cancer. N Engl J Med 354: 270-282, 2006.

8. McDonnell DP, Park S, Goulet MT, Jasper J, Wardell SE, Chang CY, Norris JD, Guyton JR and Nelson ER: Obesity, cholesterol metabolism and breast cancer pathogenesis. Cancer Res 74: 4976-4982, 2014.

9. Zhang J, Lu JP, Suter DM, Krause KH, Fini ME, Chen B and Lu Q: Isoform- and dose-sensitive feedback interactions between paired box 6 gene and delta-catenin in cell differentiation and death. Exp Cell Res 316: 1070-1081, 2010.

10. Zhang X, Huang CT, Chen J, et al: Pax6 is a human neuroectoderm cell fate determinant. Cell Stem Cell 7: 90-100, 2010.

11. Shyr CR, Tsai MY, Yeh S, Kang HY, Chang YC, Wong PL, Huang $\mathrm{CC}$, Huang KE and Chang C: Tumor suppressor PAX6 functions as androgen receptor co-repressor to inhibit prostate cancer growth. Prostate 70: 190-199, 2010.

12. Muratovska A, Zhou C, He S, Goodyer P and Eccles MR: Paired-Box genes are frequently expressed in cancer and often required for cancer cell survival. Oncogene 22: 7989-7997, 2003. 
13. Moelans CB, Verschuur-Maes AH and van Diest PJ: Frequent promoter hypermethylation of BRCA2, CDH13, MSH6, PAX5, PAX6 and WT1 in ductal carcinoma in situ and invasive breast cancer. J Pathol 225: 222-231, 2011.

14. Zong X, Yang H, Yu Y, Zou D, Ling Z, He X and Meng X: Possible role of Pax- 6 in promoting breast cancer cell proliferation and tumorigenesis. BMB Rep 44: 595-600, 2011.

15. Sobin LH and Wittekind CH: TNM Classification of Malignant tumours. 6th edition. John Wiley \& Sons, Hoboken, NJ, USA, 2002.

16. Zhang B, Cao X, Liu Y, et al: Tumor-derived matrix metalloproteinase-13 (MMP-13) correlates with poor prognoses of invasive breast cancer. BMC Cancer 8: 83, 2008.

17. Lang D, Powell SK, Plummer RS, Young KP and Ruggeri BA: PAX genes: Roles in development, pathophysiology, and cancer. Biochem Pharmacol 73: 1-14, 2007.

18. Yang Q, Shao Y, Shi J, Qu Y, Wu K, Dang S, Shi B and Hou P: Concomitant PIK3CA amplification and RASSF1A or PAX6 hypermethylation predict worse survival in gastric cancer. Clin Biochem 47: 111-116, 2014.

19. Yao D, Shi J, Shi B, et al: Quantitative assessment of gene methylation and their impact on clinical outcome in gastric cancer. Clin Chim Acta 413: 787-794, 2012.
20. Selvarajah S, Pyne S, Chen E, et al: High-resolution array CGH and gene expression profiling of alveolar soft part sarcoma. Clin Cancer Res 20: 1521-1530, 2014.

21. Mascarenhas JB, Young KP, Littlejohn EL, Yoo BK, Salgia R and Lang D: PAX6 is expressed in pancreatic cancer and actively participates in cancer progression through activation of the MET tyrosine kinase receptor gene. J Biol Chem 284: 27524-27532, 2009.

22. Zhou YH, Wu X, Tan F, et al: PAX6 suppresses growth of human glioblastoma cells. J Neurooncol 71: 223-229, 2005.

23. Zhou YH, Tan F, Hess KR and Yung WK: The expression of PAX6, PTEN, vascular endothelial growth factor, and epidermal growth factor receptor in gliomas: relationship to tumor grade and survival. Clin Cancer Res 9: 3369-3375, 2003.

24. Chen L, Romond E, Chokshi S, Saeed H, Hodskins J, Stevens M, Pasley G, Weiss $\mathrm{H}$ and Massarweh S: A prognostic model of early breast cancer relapse after standard adjuvant therapy and comparison with metastatic disease on initial presentation. Breast Cancer Res Treat 136: 565-572, 2012. 